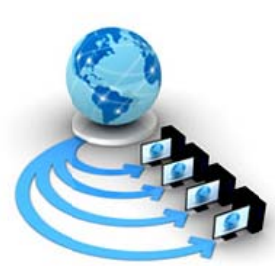

Volume 9, No. 1, January-February 2018

International Journal of Advanced Research in Computer Science

RESEARCH PAPER

\title{
A CASE STUDY ONHGNNA BASED PREDICTION OF RESONANT FREQUENCY FOR SQUARE LOOP FREQUENCY SELECTIVE SURFACE
}

\author{
Mahuya Panda ${ }^{1}$ andParthaPratim Sarkar ${ }^{2}$ \\ ${ }^{1}$ Dept. of Electrical Engineering, Camellia school of Engineering and Technology, Kolkata-124 \\ ${ }^{1,2}$ Department of Engineering and Technological Studies \\ University of Kalyani, Kalyani, Nadia, -741235, West Bengal, India
}

\begin{abstract}
Frequency Selective Surface is a well accredited scientific tag in present day communication engineering. Investigations on Frequency Selective Surface applications haveexhibited sharp inclination of research interest among researchers worldwide. In due course of action several new softwarefor Frequency Selective Surface simulationwere reported, but they failed to furnish satisfactory results in terms of computational complexity, in simulation related time consumption or even in accuracy. In order to sustain the progression of Frequency Selective Surface research the authors experienced the earnestness to deploy soft computing tool for simulation of different Frequency Selective Surface structures. Accordingly, in their earlier research attempts the authors demonstrated how Genetic Algorithm or Particle Swarm Optimization can be articulated to train the Artificial Neural Network for the simulation of Frequency Selective Surface. Here the authors tender a hybridized algorithm to amplify the easiness of simulating Frequency Selective Surface square loop structure. The authors humbly admit that the results are immensely satisfying compared to their software counterpart.
\end{abstract}

Keywords: ANN, FSS, BPA, HGNNA, GA

\section{INTRODUCTION:}

Frequency Selective Surface (FSS) is enriched with a good number of successful research attempts since its very inception. The majority of its implementation is envisaged in military applications. Yet in the last few years a strong presence of FSS is feltin commercial applications too. Concurrently researchersalso investigated FSS based wide band and multiple frequency band applications [1, 2]. It is anticipated that FSS oriented high speed communication switchable topology for Amplitude Shift Keying (ASK) Modulator [2] will substitute today's communication system in proximity. In this regard both band pass or band stop spatial filtersarebeing constructedwith FSS. More significantly FSS reduces the Radar Cross Section (RCS) [3] in such designs. Thus FSS is incorporated in satellite communication to design the reflector of antenna [4]. Beyond communication engineering, FSS ushered enormous scope in various field like smart building construction, medical detection, micro ovens \& other commercial appliances[5, 6]. Moreover, Costa et.al, used FSS for designing chip less Radio Frequency Identification (RFID) [7].Hence the vast application of FSS researches can be hardly accumulated in an ephemeral way; rather it can be convincingly stated that FSS is venturing new research initiatives by involving researchers in other fields of engineering.

Ample study on the application of FSS involves Band Width (BW) and Resonant Frequency $\left(\mathrm{R}_{\text {freq }}\right)$ of a structure. More often FSS depends on the size, shape, periodicity, width and dielectric permeability. To obtain the maximum performance of FSS the parameter extraction of FSS is absolutely essential. Generally, FEKO and ANSOFT like commercial software are used for parametric extraction ofFSS. All these software are based on Method of Moment (MOM) and Finite Element Method (FEM). But the fragility is that all commercial software require high configuration of computer system, even though the simulation time consumed by the software is uncomfortably very long for any specific design.Eventually a new methodology for parametric analysis of FSS design is indispensable.

The authors hereby are in a frame of mind to launch a new approach to study the characteristic of FSS using soft computing tools. To begin withthe authors earlier introduced Artificial Neural Network (ANN) for analysis and synthesis of FSS structure in [8]. Then the authors endeavored different ANN and Particle Swarm Optimization (PSO) techniques in [9 and 10] for prediction of $\mathrm{R}_{\text {freq }}$ and periodicity of different structures.Hereby the authorstake a leap forward to introduce a novel approach to study the FSS for parametric extraction. The modus operandi here is to hybridize Genetic Algorithm (GA) to train ANN model in efficient manner so that the parametric extraction of $\mathrm{R}_{\text {freq }}$ with minimum error can be attained. Empirical resultsrevealed that inclusion of hybrid algorithm is highly appreciable as the resultsare in good agreement with commercially viable software generated results.

\section{DESIGN PARAMETRIC OF FSS:}

The proposed FSS structure is designed on FR4EPROXY dielectric. A square loop FSS structure is proposed here for prediction of resonant frequency. Dimensionally $10 \times 10 \mathrm{~mm}^{2}$ unit cells are proposed. Each unit cellcomprises of $1.6 \mathrm{~mm}$ width. Each square patch contains two concentric square loops with $1 \mathrm{~mm}$ thickness and a square patch is a $2 \mathrm{X} 2 \mathrm{~mm}^{2}$ solid patch. The proposed unit cell is depicted in figure -1. Copper is used to design the patches. As illustrated the periodicity of proposed FSS variesfrom $11 \mathrm{~mm}$ to $20 \mathrm{~mm}$ with $0.5 \mathrm{~mm}$ interval in $\mathrm{X}$ direction and Y-direction respectively. 


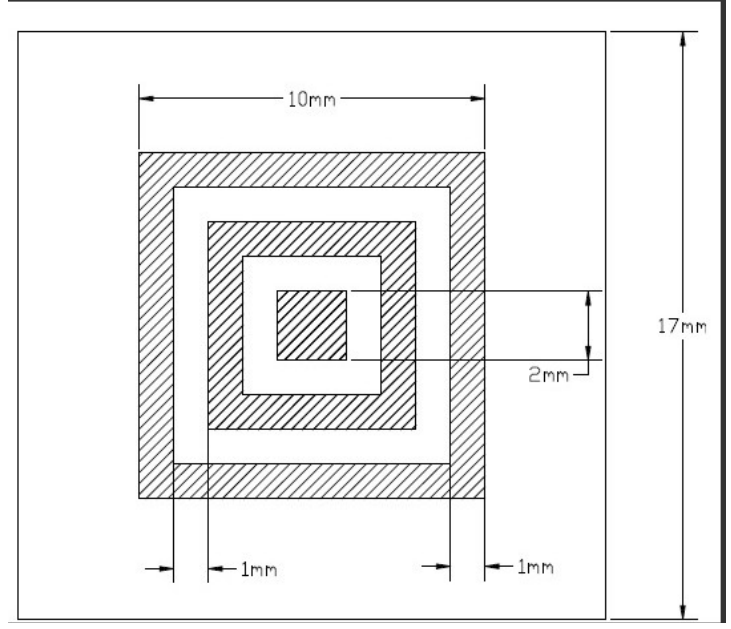

Fig.-1 Proposed unit cell Structure for FSS analysis

Here the authors obtained 250 dataset using ANSOFT simulation software which is based on MOM to train the ANN model. More conveniently this 250 dataset is used to train the proposed ANN model by GA. The programs are coded using MATLAB. The $\mathrm{R}_{\text {freq }}$ isobtained by hybridizing GA with ANN termed as Hybrid Genetic Neural Network Algorithm (HGNNA)which is unique of its kind and later on it is compared with MOM based ANSOFT result.

\section{PROPOSED CONFIGURATION OF HGNNA MODEL:}

\section{Genetic Algorithm:}

It is manifested that the traditional optimization problem is more inefficient due to computation complexity. Besides, the relative optimal solution remains in the closest range of the starting point. Obviously to continue with the finding of the global optimal solution with highest degree of probabilityresearchers are left with no choice but to indulge GA in the optimization problem [10].Be it unambiguously mentioned that GA is inspired by the Darwin's theory for survival strategy of fittest value. In short, to endeavor GA(i) reproduction, (ii) crossover and (iii) mutation named three genetic search processes are used.

\section{Artificial Neural Network:}

ANN demonstrates the basic building block of robust data modeling in soft computing [12]. It is considered for establishing the input output relationship of nonlinear domain. Basically ANN is a data analysis system inspired by human nervous organism. Analytically the ANN consists of different layers and large number of processing units. Structurally the first and last layer is the input and output layer of the system. The other layer amid the input and output layer is the hidden layer. To match up with human body each of the processing unit (neuron) are connected to the next layer through some weight. Figure-2 proposes the ANN model to be considered for HGNNA in this empirical research report.

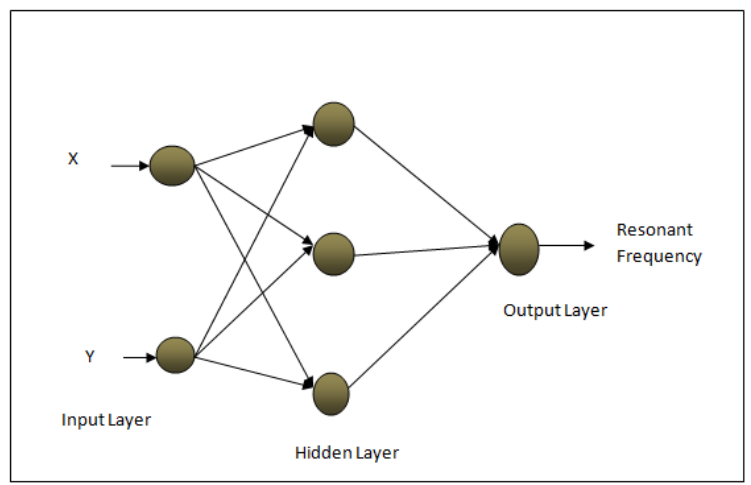

Figure-2 Proposed ANN model HGNNA simulation

\section{PROPOSED APPROACH OF ANN WEIGHT OPTIMIZATION (HGNNA):}

As mentioned earlier, this particular research work exercised GA to train the connecting weight parameter of ANN model as is shown in figure-2. The valueof GA here is its simplest implementation with less computational complexity. A Multi Layer Feed Forward (MLFF) network is taken into account for the proposed ANN model in this research work. Generally MLFF model is trained by Back Propagation Algorithm (BPA).It works on gradient decent method and adheres to the provided input data. But that's not all, BPA faces intrinsic challenges.

BPAin contrastbasesupon the solution of gradient decent steepest vector for finding the global optimum value along the error surface. Now obviously it can be confronted by any other hybrid algorithms. It is undoubtedly a fast algorithm but it is tapped in to the local minima and owing to such limitation it is unable to find the global optimum value.Thus in other words it can be stated that to overcome the above mentioned problem GA is introduced for training this weight of ANN model as GA is an efficient global searching problem. GA has increasingly scattered enough attentionbecause it has the potentiality to retain significant optimization as well as error less throughput. Being so an improved GA is adopted herein. The basis of the improved GA is that the chromosome is subsequently arranged in the central region and boundary region of the search domain just after the accomplishment of crossover. Hence the global optimum solution can be achieved for the case of next generation [13]. This is illustrated in the following. 
$\operatorname{crom}_{c}^{1}=\left[\begin{array}{llll}\operatorname{crom}_{1}^{1} & \operatorname{crom}_{2}^{1} \quad \ldots \ldots & \operatorname{crom}_{\text {novar }}^{1}\end{array}\right]=$

$\frac{P_{1}+P_{2}}{2}$.

$\operatorname{crom}_{c}^{2}=\left[\begin{array}{llll}\operatorname{crom}_{1}^{2} & \operatorname{crom}_{2}^{2} & \ldots \ldots & \operatorname{crom}_{\text {novar }}^{2}\end{array}\right]=$

$P_{\text {max }}(1-w)+\max \left(P_{1}, P_{2}\right) w \ldots \ldots \ldots \ldots \ldots(2)$

$\operatorname{crom}_{c}^{3}=\left[\begin{array}{llll}\operatorname{crom}_{1}^{3} & \operatorname{crom}_{2}^{3} & \ldots \ldots & \operatorname{crom}_{\text {novar }}^{3}\end{array}\right]=$

$P_{\min }(1-w)+\min \left(P_{1}, P_{2}\right) w \ldots \ldots \ldots \ldots \ldots . .(3)$

$\operatorname{crom}_{c}^{4}=\left[\begin{array}{llll}\operatorname{crom}_{1}^{4} & \operatorname{crom}_{2}^{4} & \ldots \ldots & \text { crom }_{\text {novar }}^{4}\end{array}\right]=$

$\frac{\left(P_{\max }+P_{\min }\right)(1-w)\left(P_{1}+P_{2}\right)}{2} \ldots \ldots$ (4)

Here the chromosomes of next generation are represented bycrom $_{c}^{1} \sim$ crom $_{c}^{4}$. Chromosomes are chosen from the parent $\mathrm{P}_{1}$ and $\mathrm{P}_{2}$ respectively. Genes in the two chromosomes $\mathrm{P}_{1}$ and $\mathrm{P}_{2}$ are represented by $\max \left(\mathrm{P}_{1}, \mathrm{P}_{2}\right)$ and $\min \left(\mathrm{P}_{1}, \mathrm{P}_{2}\right)$ respectively.

$P_{\text {min }}=$

$\left[\begin{array}{llll}p r n t_{\min }^{1} & \text { prnt }_{\min }^{2} & \cdots \cdots & \text { prnt }_{\min }^{\text {novar }}\end{array}\right]$.

..................(5)

$P_{\max }=$

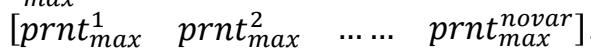

Accordingly,

the

$\mathrm{i}^{\text {th }}$ parentgenes

arerepresentedbyprnt $t_{\text {min }}^{i}, p r n t_{\text {max }}^{i}$. Thevalue of parameter ' $w$ ' is chosen randomly between $(0,1)$. Using the equation (1) and (2) new set chromosomesare generatedwhich are later oncirculated in the central region of search region. Simultaneously, equation (3) and (4) generated thechromosomes among boundary region.

Again it must be considered that Gradient decent method highly depends on the structure of ANN model whereas the proposed HGNNA is highly independent of any proposed structure.ANN weight parameters are formed depending on the variations of the GA. For cost function of GA the authors deliberated on different Root Means Square Error (RMSE) criterions. The true objective of the proposed algorithm lies in finding the optimal value with minimum error value.

\section{RESONANT FREQUENCY ACCURACY EVOLUTION:}

The authors took severe pain to obtain accuracy in measurement and subsequently different error criterions like Mean Absolute Percentage Error (MAPE), the Sum Square Error (SSE), the Root Mean Squared Error (RMSE) and Standard Deviation of Error (SDE) are incorporated in the operation. These error criterionsare used as a function of simulated Resonant Frequency using HGNNA.Followings are the above mentioned error criterions -

$$
\begin{aligned}
& M A P E= \\
& \frac{100}{N} \sum_{h=1}^{N} \frac{\left(R_{\text {freq }}^{a}-R_{\text {freq }}^{f}\right)}{\overline{R_{\text {freq }}}{ }_{h}^{a}} \\
& S S E=\sum_{h=1}^{N}\left(R_{\text {freq }_{h}}^{a}-\right. \\
& \left.R_{\text {freq }}^{f}\right)^{2} \\
& R M S E= \\
& \sqrt{\frac{1}{N} \sum_{h=1}^{N}\left(R_{\text {freq }}^{a}-R_{\text {freq }}^{f}\right)^{2}} .
\end{aligned}
$$

$$
\bar{e}=\frac{1}{N} \sum_{h=1}^{N} e_{h}, \quad \begin{aligned}
& f \\
& R_{\text {freq }_{h}}^{f} .
\end{aligned}
$$

$R_{\text {freq }}{ }_{h}^{a}$ is actual resonant frequency.

$R_{\text {freq }} f_{h}$ is predicted resonance frequency.

Here $\mathrm{N}$ is the number of data set.

\section{RESULT AND DISCUSSION:}

The main focus of this research work is pinpointed towards obtaining the resonant frequency with minimum error via soft computing tool. HGNNA stands to be a novel approach to mobilize a soft computing tool for prediction of resonant frequency. Here the authorshave generated 250 dataset using ANSOFT simulation software for prediction of resonant frequency. After generating the dataset authors used 240 dataset for ANN training using the proposed HGNNA.Remaining 10 data setsare used for validation of trained ANN model.For better clarity of result two algorithms are accumulated here to form one hybrid algorithm in order to availerrorless result. ANN weight is optimized by GA to obtainthe predicted resonant frequency with least error.All programsare written in MATLAB.Later on the HGNNA based resultsarecomparedto the MOM based ANSOFT simulation result and are lastly accumulated

\begin{tabular}{|c|c|c|c|c|}
\hline \multirow{3}{*}{$\begin{array}{l}\text { Sl. } \\
\text { No }\end{array}$} & \multicolumn{2}{|c|}{ Input Data } & \multicolumn{2}{|c|}{ Out Put Data } \\
\hline & X- & $\mathrm{Y}-$ & MOM & HGNNA \\
\hline & $\begin{array}{l}\text { direction } \\
\text { periodicity } \\
\text { (mm) }\end{array}$ & $\begin{array}{l}\text { direction } \\
\text { periodicity } \\
(\mathrm{mm})\end{array}$ & $\begin{array}{c}\text { Resonant } \\
\text { Frequency } \\
\text { GHz }\end{array}$ & $\begin{array}{c}\text { Resonant } \\
\text { Frequency } \\
\text { GHz }\end{array}$ \\
\hline 1 & 12 & 12 & 5.76 & 5.83 \\
\hline 2 & 14.5 & 13 & 6.05 & 5.96 \\
\hline 3 & 14 & 14 & 6.17 & 6.20 \\
\hline 4 & 15.5 & 14 & 6.17 & 6.19 \\
\hline 5 & 12 & 16 & 6.26 & 6.25 \\
\hline 6 & 11 & 11 & 5.29 & 5.31 \\
\hline 7 & 12.5 & 14.5 & 6.18 & 6.12 \\
\hline
\end{tabular}
in Table-I. Seemingly all results are quite in good agreement. Rendering upon the tabulated datathe convergences of HGNNA are shown in figure 3.

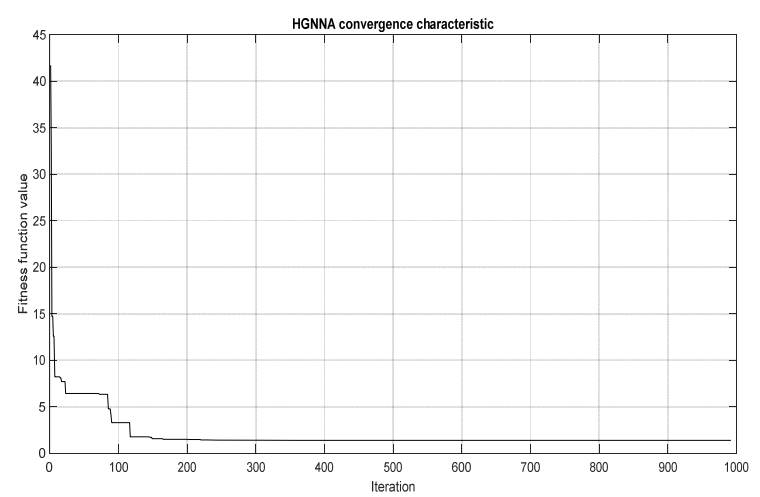

Figure3: RMSE for output in HGNNA(ANN trained by GA) Table-I Predicted result for Resonant Frequency HGNNA (ANN trained by GA)

$\sqrt{\frac{1}{N} \sum_{h=1}^{N}\left(e_{h}-\bar{e}\right)^{2}}$

$S D E=$ 


\section{CONCLUSION}

It is perceived that FSS based research yet possess a great dearth. Bringing down FSS from research labs to consumable electronics is a fascinating but challenging maneuver. In this regard few software are categorically reported but the fact is that such software are very time consuming and less efficient and demands very high speed computer configuration. Thus the computational complexity has to be eased effectively. Research attempts in this regard have been prioritized since the beginning of this decade. The authors earlier have deployed GA as well as PSO to train the ANN model. Being motivated by the degree of success the authors hereby made another effort by hybridizing GA with ANN. Ample studies of square loop FSS model are pondered over for simplification and easy understanding. Here HGNNA is used for prediction of resonant frequency of square loop FSS. The results are validated using two or three set of data and as evident from the tabulated data the results are in good accord. Due to independency of structure of ANN model HGNNA have edgeover other conventional topologies in training of ANN.Additionally with the success of HGNNA, the authors are persistent enough to use similar or different soft computing algorithms for different FSS structures in near future, only to exploit FSS for the sake of everyday mankind.

\section{REFERENCE}

[1] B Munk , “ Frequency Selective Surfaces: Theory and Design “(NewYork: John Wiley \& Sons Inc.), 2000.

[2] N. Guerin ; C. Hafner ; X. Cui ; R. Vahldieck, "Compact directive antennas using frequency-selective surfaces (FSS)", Microwave Conference Proceedings, APMC 2005. Asia-Pacific Conference Proceedings, vol.-1, pp 4, 2005,.

[3] Ortiz, J. D., Baena, J. D., Marques, R., et al., "A bandpass/stop filter made of SRRS and C-SRRS". In Antennas and Propagation (APSURSI), 2011 IEEE International Symposium on, 2011.
[4] Ghaffer I.Kiani and Rabah W. Aldhaheri, "Wide Band FSS for Increased Thermal and Communication Efficiency in Smart Buildings”, IEEE, pp 2064-2065, 2014.

[5] Woo Cheol Choi, Ki Joon Kim, Young JoongYoon , "Design of FSS Unit-cell Integrated in Water Bolus for Microwave Biomedical Application” Proceedings of iWEM2014, Sapporo, Japan, 2014, pp 265 - 266.

[6] Ghaffer I. Kiani and Rabah W. Aldhaheri,” Wide Band FSS for Increased Thermal and Communication Efficiency in Smart Buildings”, IEEE, pp-2064-2065, 2014.

[7] Filippo Costa, Simone Genovesi, AgostinoMonorchio," Chipless RFID Trasponders by using Multi-resonant High-Impedance Surfaces”, Proceedings of the 2013 International Symposium on Electromagnetic Theory, pp 401-403, 2013.

[8] Mahuya Panda, PoulamiSamaddar, P. P. Sarkar," Artificial Neural Network for the Analysis and Design of a Frequency Selective Surface with SLITS”, International Journal of Computer Applications in Engineering Sciences, pp 81-83,2013

[9] M Panda, S Nandi and P P Sarkar, “A comparative study of performance of different back-propagation neural network methods for prediction of resonant frequency of a slot-loaded double-layer frequency-selective surface", Indian Journal of Physics, 2015, Volume 89, Issue 12, pp.1283-1286.

[10] Mahuya Panda and ParthaPratim Sarkar, "Prediction Of Periodicity Of FSS Structure Using Particle Swarm Optimization”, I-manager's Journal on Electronics Engineering, vol. 7 no. 3 ,pp25-31, 2017.

[11] David. E. Goldberh," Genetic Algorithms in Search, Optimization, and Machine Learning”, Addison-Wesley Professional; 1 edition, 1989.

[12] S Haykin, "Neural Networks, A Comprehensive Foundation”,(Englewood Cliffs: Prentice Hall) 2nd edn, 1999.

[13] Frank H. F. Leung, H. K. Lam, S. H. Ling, and Peter K. S. Tam," Tuning of the Structure and Parameters of a Neural Network Using an Improved Genetic Algorithm”, IEEE TRANSACTIONS ON NEURAL NETWORKS, VOL. 14, NO. 1, pp 79-88,JANUARY 2003 